\title{
Early Seed Development in Wheat $\times$ Rye Crosses Following Pollination with Irradiated Rye Pollen
}

\author{
F. Ahmad and G. J. Scoles \\ Dept. of Crop Science and Plant Ecology, University of \\ Saskatchewan, Saskatoon, Saskatchewan, Canada S7N OWO
}

Accepted March 28, 1988

In recent years irradiated pollen induced gene transfer experiments have met with varying degrees of success (Pandey 1975, Davies 1981, Ahmad et al. 1983, Snape et al. 1983). In our own experiments to transfer genes from rye to wheat, we could successfully rescue immature embryos from lower gamma radiation treatments (2-5 Gy) but not from the higher radiation (10-50 Gy) treatments (Ahmad et al. 1983). We, thus, decided to study the effect of rye pollen irradiation on the developing embryo and endosperm in wheat $\times$ rye crosses, when such pollen was used for pollination.

There have been only a few previous reports of the effect of irradiated gametes on the developing embryo and endosperm. In a study with Lycopersicum pimpinellifolium, Nishiyama and Uematsu (1967) consistently noted reduced growth rates of embryo and endosperm in zygotes fertilized by irradiated pollen. At 50 and $100 \mathrm{~Gy}$ of X-ray exposure, zygote formation or growth of the embryo and endosperm was almost completely inhibited. Various mitotic aberrations were observed. In similar studies with Lilium (Cave and Brown 1954) and wheat (Snape et al. 1983), embryo and endosperm cells were characterized by bridges, fragments, lagging chromosomes, micronuclei and necrotic nuclei. In wheat (Snape et al. 1983), no qualitative differences were observed between a 2 Gy and a 5 Gy X-ray treatment, but the frequency of aberrations was directly related to the radiation dose. In Drosophila melanogaster, Sonnenblick (1940) observed that the differentiation of the embryos containing one set of X-rayed chromosomes, from either the sperm or the egg, was disturbed to the extent that they were sometimes only structureless masses of cells. All of the above mentioned studies involve intraspecific crosses, however, the present report deals with such studies in an intergeneric cross.

\section{Materials and methods}

Plants of a hexaploid spring wheat line "JBT-3", having high crossability with rye, and a diploid spring rye cultivar "Gazelle" were grown in a glasshouse under natural daylight supplemented with artificial fluorescent light to provide an illumination intensity of $500 \mu \mathrm{E} . \mathrm{m}^{-2}$. $\mathrm{s}^{-1}$ and a $16 \mathrm{hr}$ day length with temperature ranging between 18 and $25^{\circ} \mathrm{C}$. Rye pollen was collected in glass petri plates and irradiated in a gamma cell $\left({ }^{\circ}{ }^{\circ} \mathrm{Co}\right.$ source) at an exposure rate of $476 \mathrm{rad} \mathrm{min}^{-1}$. The duration of irradiation was adjusted in such a way that pollen grains received radiation exposure of $2,5,10,15$, and $50 \mathrm{~Gy}$. Unirradiated $(0 \mathrm{~Gy})$ rye pollen was used as a control. Previously emasculated wheat florets were pollinated with irradiated or control rye pollen when the stigmas were quite feathery and receptive.

For the study of early seed development, pollinated wheat pistils were harvested at 1, 2, 3, 4, 5 and 6 days after pollination (DAP) and fixed immediately in Farmer's fixative ( 3 parts $95 \%$ ethyl alcohol : 1 part glacial acetic acid, $v / v$ ). The rest of the procedure adopted was as suggested by Bennett et al. (1975), except that staining of the embryo sacs was done in Schiff's reagent. Temporary slides were prepared to determine if fertilization had occurred and, if 
so, to determine the extent of post-fertilization development. To ascertain the embryo shape and the distribution of endosperm nuclei and antipodal cells, the cover slips were not tapped. The cover slips were, however, subsequently tapped very gently to facilitate spreading of the cells for counts of the number of cells (or nuclei) in the embryo and endosperm. Cells (or nuclei) at all stages of division, other than late telophase when distinct nuclei were formed, were counted as one cell (or nucleus).

\section{Results and discussion}

The number of cells in the embryo at the six harvest dates are shown in Fig. 1. In all treatments embryo growth followed a similar pattern, however, the growth rates were different. Soon after fertilization the zygote entered a resting stage and the first division did not occur about 24 hrs after pollination. The exact timing of the first zygotic division in the irradiated treatments was difficult to ascertain because it presumably fell in between day 1 and 2 . How-

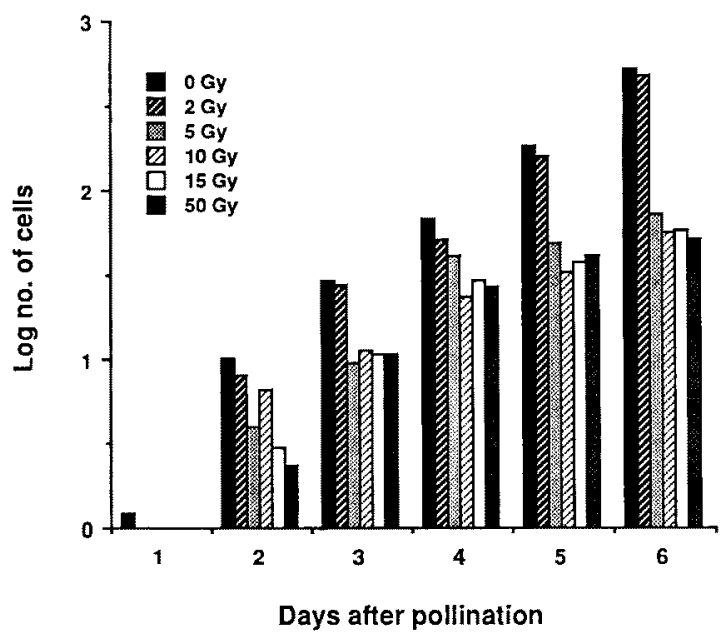

Fig. 1. Logarithmic number of cells per embryo at 1 to 6 days after pollination of "JBT-3" wheat pistils with irradiated "Gazelle" rye pollen.

ever, from the extent of chromatin condensation it seemed that pollen irradiation delayed the first zygotic division by at least a few hours compared to the controls. The embryo growth rate in the irradiated materials was slower than that in the controls (Fig. 1). At the fifth and the sixth DAP there were two distinct growth rates, that of the 2 Gy and the control treatments and that of the higher radiation treatments (Fig. 1).

The 2 Gy treatment fruits had much less endosperm tissue than the control fruits while the higher radiation fruits had none at the time of embryo culture (11 DAP). Data for the development of one to three-day old endosperms for the various treatments are shown in in Fig. 2. Endosperm nuclei counts could not be made any later than 3 DAP in the control and 2 Gy treatments beacause of the accumulation of starch grains which masked the nuclei. In the higher radiation treatments, the degeneration of the endosperm nuclei began 3 DAP and the endosperm nuclei would not stain. Endosperm mitosis commenced before the first DAP in all treatments. At this harvest date the primary endosperm nucleus had already undergone three or four division cycles. The initial divisions were found to be nearly synchronous up to the first harvest date after which synchrony was lost. 


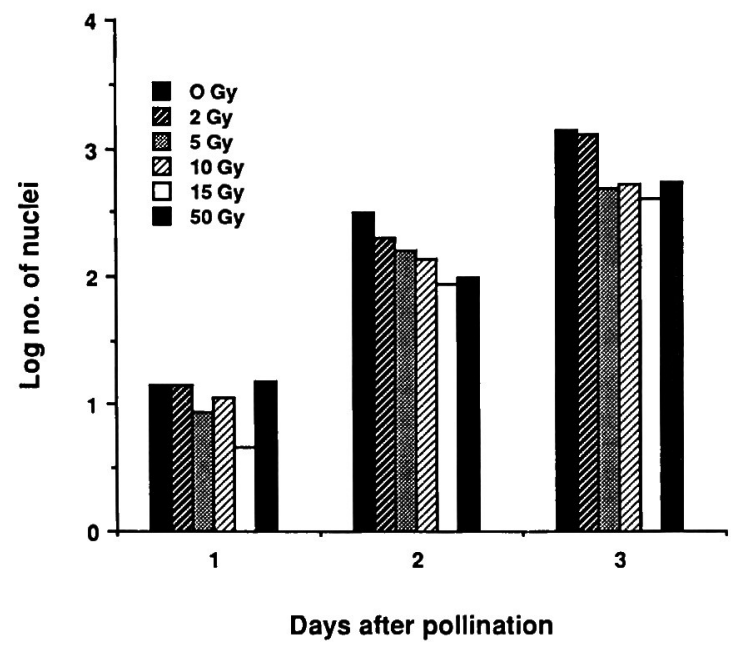

Fig. 2. Logarithmic number of nuclei per endosperm at 1 to 3 days after pollination of "JBT-3" wheat pistils with irradiated "Gazelle" rye pollen.
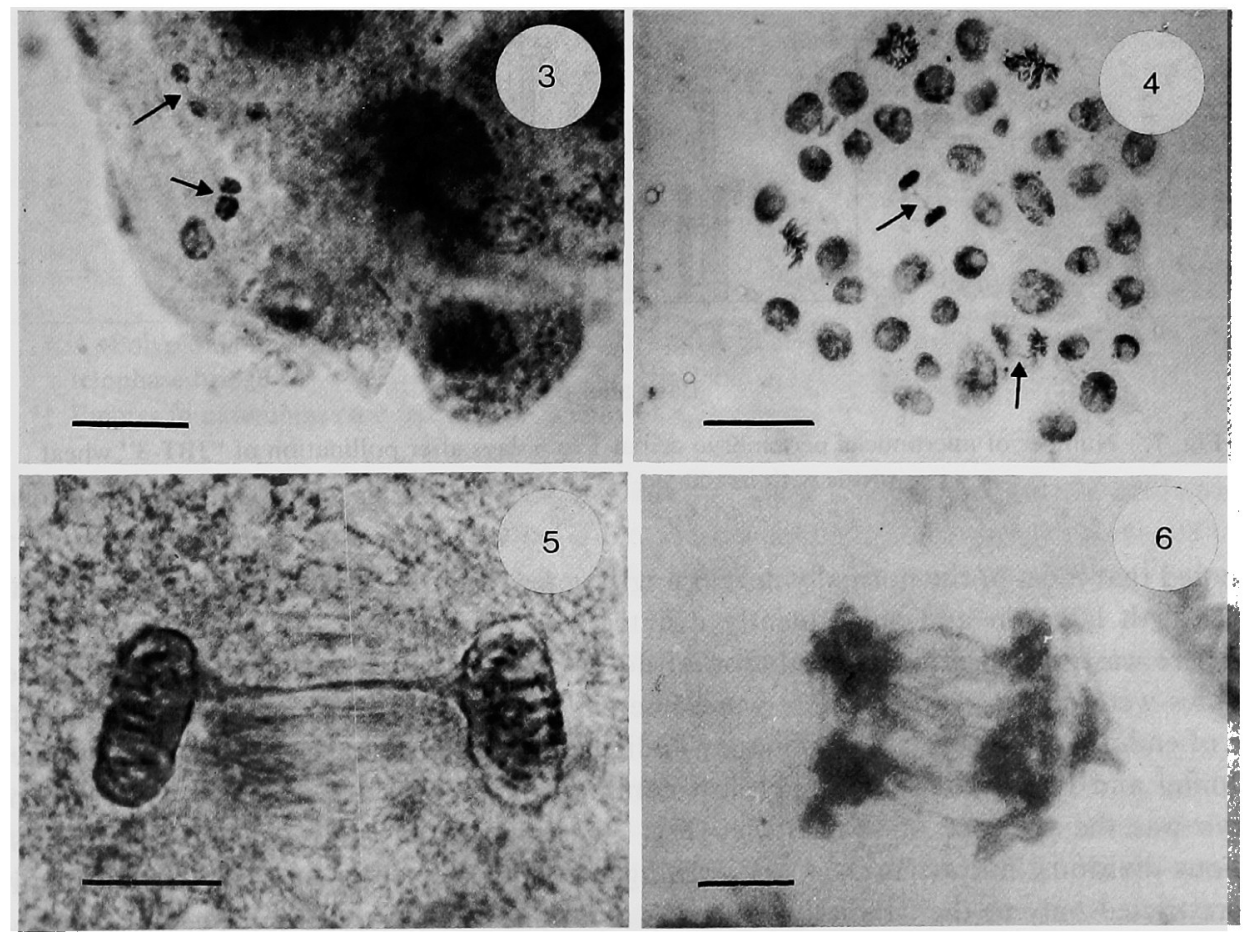

Figs. 3-6. Early seed development when "JBT-3" wheat pistils were pollinated with irradiated "Gazelle" rye pollen. 3, part of a three-day old embryo from the $10 \mathrm{~Gy}$ treatment showing micronuclei (arrows). 4, a six-day old embryo from the $50 \mathrm{~Gy}$ treatment showing asynchronous divisions and mitotic bridges (arrows). 5, telophase bridge in a two-day old endosperm from the 15 Gy treatment. 6, a large polyploid nucleus undergoing division in a two-day old endosperm from the $50 \mathrm{~Gy}$ treatment. Bar in Figs. 3 and 4 represent 15 and $50 \mu \mathrm{m}$, respectively, while in Figs. 5 and 6 represent $10 \mu \mathrm{m}$. 
The number of endosperm nuclei was quite variable among the treatments. It was maximum around $3 \mathrm{DAP}$, for the $5 \mathrm{~Gy}$ and higher radiation treatments, which was about the time when endosperm cellularization initiated. The slower growth rate of endosperm from the 2 Gy treatment compared to that for the control, noticed on day 2, was no longer observed at 3 DAP (Fig. 2). The differences in nuclear division rates can be seen at the third harvest date when the control and the 2 Gy treatments had two to three times more number of nuclei than the other higher radiation treatments. An irradiation dose of $5 \mathrm{~Gy}$ or more, did not decrease the endosperm growth rate any further.

The effect of higher radiation exposure $(5-50 \mathrm{~Gy})$ was to greatly reduce the growth rates of the embryo and endosperm while growth rate in the $2 \mathrm{~Gy}$ fruits was almost the same as that of the control. These tissues collapsed and degenerated by 6 DAP in the higher radiation (5$50 \mathrm{~Gy}$ ) fruits. The result was shrivelled seeds without either embryo or endosperm. Delayed and reduced growth rate in the two hybrid tissues, following pollination with irradiated pollen, has also been reported in Lycopersicum pimpinellifolium (Nishiyama and Uematsu 1967). It

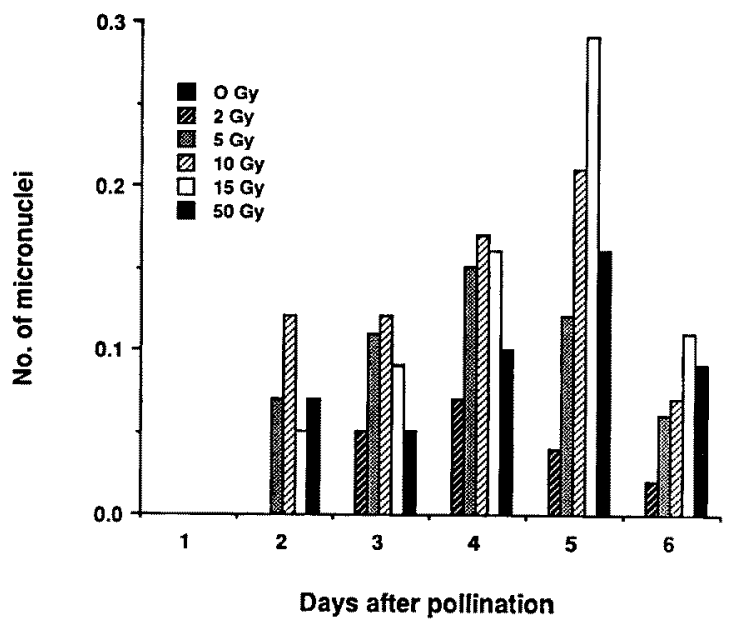

Fig. 7. Number of micronuclei per embryo cell at 1 to 6 days after pollination of "JBT-3" wheat pistils with irradiated "Gazelle" rye pollen.

was noted that most of the normally developing zygotes from the higher radiation doses ceased their growth later on and subsequently disintegrated (Nishiyama and Uematsu 1967).

There was no indication of inhibition of double or triple fusion, however, a few rare embryo sacs were observed where there was some embryo growth without a corresponding presence of endosperm or vice versa. Similar findings have also been reported in Triticum durum by Donini and Hussain (1968). The most common abnormality observed in the developing embryo was the presence of micronuclei (Fig. 3). Embryos were also found to show asynchronous divisions, mitotic bridges and lagging chromosomes (Fig. 4). These abnormalities were restricted only to the irradiated materials and none was observed in the control embryos. The number of micronuclei per embryo cell did not reveal any specific patterns in terms of increasing radiation levels (Fig. 7). However, the highest proportion of micronuclei happened to fall during the period of 3,4 and $5 \mathrm{DAP}$ in various radiation treatments which is about the time when the endosperm showed degeneration. The very slow growth rate of embiyo, starting around 3 DAP (Fig. 1) also indicated degeneration of embryo cells.

The kind of nuclear abnormalities seen in the endosperm was much greater than those 
observed in the embryo. Micronuclei were never observed in the endosperm as opposed to the embryo, where this was the major abnormality. Other abnormalities exhibited by the endosperm tissue were non-congressional chromosomes, lagging chromosomes, anaphase and telophase bridges, dumbell nuclei, polyploid nuclei and necrotic nuclei (Figs. 5, 6). Various types of mitotic aberrations observed in this study, were also observed in previous and related reports (Sonnenblick 1940, Cave and Brown 1954, Snape et al. 1983). Even the control endosperms were not devoid of these abnormalities although the frequency of aberrant nuclei were very low (Table 1). Degeneration of the endosperm in the 5 Gy and higher radiation treatments became very pronounced at 3 DAP. Another abnormality noticed was that endosperm nuclei were either faintly or not stained at all, suggesting that DNA was probably being broken down.

At higher levels of gamma radiation it is expected that many chromosomes would be broken. Some of the broken ends might unite to form dicentrics. When male nuclei, with such chromosome aberrations, fuse with the egg or polar nuclei, brigdes would be expected at the following anaphase. This was observed in the present study. Mitotic bridges in the endosperm of wheat $\times$ rye hybrids have also been attributed to the presence of late replicating telomeric rye heterochromatin (Bennett 1977) and wheat chromatin (Varghese and Lelley 1983). Nucleo-cytoplasmic interactions and the general disorganization caused by the pre-

Table 1. Ranges in the frequency of aberrant nuclei per endosperm at 1 to 3 days after pollination (DAP) of "JBT-3" wheat with irradiated (0-50 Gy) "Gazelle" rye pollen

\begin{tabular}{|c|c|c|c|c|}
\hline \multirow{2}{*}{ DAP } & \multicolumn{4}{|c|}{ Frequency of aberrant nuclei* } \\
\hline & A & B & $\mathrm{C}$ & D \\
\hline 1 & $\begin{array}{c}00-.14 \\
(00)^{* *}\end{array}$ & $\begin{array}{c}00-00 \\
(00)\end{array}$ & $\begin{array}{c}00-.12 \\
(00)\end{array}$ & $\begin{array}{l}00-.11 \\
(.03)\end{array}$ \\
\hline 2 & $\begin{array}{l}.03-.10 \\
(.02)\end{array}$ & $\begin{array}{l}.02-.06 \\
(.01)\end{array}$ & $\begin{array}{l}.03-.06 \\
(.02)\end{array}$ & $\begin{array}{l}.04-.08 \\
(.02)\end{array}$ \\
\hline 3 & $\begin{array}{l}.03-.10 \\
(.02)\end{array}$ & $\begin{array}{c}.02-.06 \\
(.01)\end{array}$ & $\begin{array}{c}.03-.10 \\
(.01)\end{array}$ & $\frac{.06-.10}{(.04)}$ \\
\hline
\end{tabular}

* A: Polyploid nuclei, B: Dumbell nuclei, C: Nuclei with lagging chromosomes, D: Nuclei with anaphase/ telophase bridges.

** Figures in parentheses are frequencies in control endosperms.

sence of rye chromatin in wheat cytoplasm could also contribute to the mitotic aberrations in the developing tissue. All these factors could further aggravate the situation in addition to the effects of gamma radiation. Thus a number of factors could be contributing to the aberrations observed in this study.

The frequency of bridges in the endosperm was quite high as compared to only a few in the embryo. This suggests that some repair had occurred in the embryo. Some reports suggest that broken chromatid ends can permanently heal in maize embryos but not the endosperms (Clark and Copeland 1940, McClintock 1941). Similar phenomenon possibly happens in the wheat $\times$ rye hybrid embryos in this study. In addition, the long resting period between fertilization and the first zygotic division might have given the broken rye chromosomes an opportunity to heal as suggested by Clark and Copeland (1940). The longer cell doubling time in the embryo, as compared to the endosperm (Bennett et al. 1975), could also be an important factor in the subsequent repair of the damaged male chromatin.

\section{Summary}

Early seed development was studied in wheat $\times$ rye crosses, in which irradiated $(0-50 \mathrm{~Gy})$ 
rye pollen was used for pollination. The number of cells and nuclei in the embryos and endosperms were scored daily for 6 and 3 days after pollination (DAP), respectively. Embryo and endosperm growth rates in the 2 Gy treatment were similar to the control (no irradiation). In other treatments, pollen irradiation greatly reduced the growth rates in both the tissues. The 2 Gy and control ovules had 7 to 10 times more embryo cells and 2 to 3 times more endosperm nuclei than the other treatments at 6 and 3 DAP, respectively. Several abnormalities were observed in the developing ovules of pistils pollinated with irradiated pollen. Degeneration of the endosperm began $3 \mathrm{DAP}$ in the $5 \mathrm{~Gy}$ and higher radiation treatments but later in the 2 Gy treatment. Embryo abortion also occurred at a later stage in the 2 Gy treatment than in the higher radiation treatments. These studies indicate that gamma radiation doses beyond 2 Gy are quite deleterious to the developing ovule and in most cases the embryo sac succumbs to higher radiation treatments.

Key wards: Pollen irradiation, wheat, rye, embryo, endosperm

\section{Acknowledgements}

The authors thank Dr. Brian P. Forster for sharing his expertise. This research was funded by a grant to GJS from Canada Department of Agriculture.

\section{References}

Ahmad, F., Scoles, G. J. and Thomas, J. B. 1983. Attempts to induce gene transfer in wheat $\times$ rye crosses by irradiation of rye pollen, The Genetics Society of Canada Bulletin 14(2): 46.

Bennett, M. D. 1977. Heterochromatin, aberrant nuclei and grain shrivelling in wheat-rye genotypes. Heredity $39: 411-419$.

-, Smith, J. B. and Barclay, I. R. 1975. Early seed development in the Triticeae. Phil. Trans. Roy. Soc. London Ser. B. 272: 199-227.

Cave, M. S. and Brown, S. W. 1954. The detection and nature of dominant lethals in Lilium. II. Cytological abnormalities in ovules after pollen irradiation. Amer. J. Bot. 41: 469-483.

Clark, F. J. and Copeland, F. C. 1940. Chromosome aberrations in the endosperm of maize. Amer. J. Bot. 27: 247-251.

Davies, R. 1981. Gene transfer in plants. Nature 291: 531-532.

Donini, B. and Hussain, S. 1968. Development of the embryo in Triticum durum following irradiation of male or female gamete. Rad. Bot. 8: 289-295.

McClintock, B. 1941. The stability of broken ends of chromosomes of Zea mays. Genetics 26: 234-282.

Nishiyama, I. and Uematsu, S. 1967. Radiobiological studies in plants. XIII. Embryogenesis following Xirradiation of pollen in Lycopersicum pimpinellifolium. Rad. Bot. 7: 481-489.

Pandey, K. K. 1975. Sexual transfer of specific genes without gametic fusion. Nature 256: 310-313.

Snape, J. W., Parker, B. B., Simpson, E. and Ainsworth, C. C. 1983. The use of irradiated pollen for differential gene transfer in wheat (Triticum aestivum). Theor. Appl. Genet. 65: 103-111.

Sonnenblick, B. P. 1940 . Cytology and development of the embryos of X-rayed adult Drosophila melanogaster. Proc. Nat. Acad. Sci. USA. 26: 373-381.

Varghese, J. P. and Lelley, T. 1983. Origin of aberrations and seed shrivelling in triticale: a re-evaluation of the role of C-heterochromatin, Theor. Appl. Genet. 66: 159-167. 